\title{
escrito poŕn..
}

\section{${ }^{1}$ Marinilda Rivera-Díaz}

Profesora del Programa Doctoral en Determinantes Sociales de la Salud de la Escuela Graduada de Salud Pública de la Universidad de Puerto Rico. Comisionada de Educación para la región de América Latina y el Caribe de la Federación Internacional de Trabajo Social. Coordinadora del Grupo de Trabajo Salud Internacional y Soberanía Sanitaria del Consejo Latinoamericano de las Ciencias Sociales.

$\triangle$ marinilda.riveradiaz@upr.edu

(DD ORCID ID https://orcid.org/0000-0002-6104-5072

Cómo citar / citation: Rivera-Díaz, M. (2020). A despatologizar y humanizar la salud mental desde las instituciones académicas: propuestas para un accionar ético político. Voces desde el Trabajo Social, 8(1), 150-175.

https://doi.org/10.31919/voces.v811.222

Recibido / received: 28 de julio de 2020
Revisado / reviewed: 27 de Noviembre de 2020
Aceptado / accepted: 8 de diciembre de 2020

Derechos de autoría / Copyright: C 2020 Rivera-Díaz, M. Este es un artículo de acceso abierto y distribuido bajo los términos de la licencia y políticas de Creative Commons Attribution 4.0 International License 


\section{A DESPATOLOGIZAR Y HUMANIZAR LA SALUD MENTAL DESDE LAS INSTITUCIONES ACADÉMICAS: PROPUESTAS PARA UN ACCIONAR ÉTICO POLÍTICO}

Marinilda Rivera-Díaz ${ }^{1}$ (D)

f open ACCESS PeER-REVIEWED

Resumen

Este ensayo tiene como algunos de sus referentes la teoría social crítica y los derechos humanos, con particular interés en la salud y la salud mental. En el mismo expondré el marco de los derechos humanos y el derecho a la salud desde los organismos internacionales, reconociendo que existen diversas críticas y debates a su concepción. Además, analizaré el rol de la agenda global neoliberal en los derechos humanos, incluyendo la salud y salud mental y su impacto en la patologización y deshumanización de la salud mental. Por último, como profesional del trabajo social y docente investigadora me propongo compartir algunas reflexiones y recomendaciones sobre el rol ético-político de la Academia en la defensa de este derecho humano.

\section{Palabras claves}

salud mental, educación superior, derechos humanos, determinantes sociales de la salud, trabajo social 


\section{Sumario}

Introducción. Salud mental como derecho humano. La globalización neoliberal y los derechos humanos. Vulnerabilización de la salud mental ante la agenda neoliberal en los derechos humanos. Rol de la Academia en la defensa de la salud mental como derecho humano. Reflexiones finales. Referencias.

\section{[EN] TO DEPATHOLOGIZE AND HUMANIZE MENTAL HEALTH FROM ACADEMIC INSTITUTIONS: PROPOSALS FOR AN ETHICAL POLITICAL ACTION}

\section{Abstract}

This essay has as some of its references the critical social theory and the human rights framework, with a particular interest in health and mental health. In this paper, I will expose the framework of human rights and the right to health from international organizations, recognizing that there are various critics and debates about its conception. In addition, I will analyze the role of the neoliberal global agenda in human rights, including health and mental health, and its impact on the pathology and dehumanization of mental health. Finally, as a professional of social work and social researcher, I intend to share some reflections on the ethical-political role of the Academy in the defense of this human right.

\section{Keywords}

mental health, higher education, human rights, social determinants of health, social work

\section{Introducción}

Las reflexiones que aquí histórico de país bajo el control de compartiré están matizadas un régimen colonial de explotación por la subjetividad de una mujer y un sistema capitalista que toma trabajadora social, puertorriqueña, nuevas configuraciones. Parto del y procedente de un contexto socio- reconocimiento de que este contexto 
ha agudizado la desigualdad, la violación de derechos humanos, la criminalización de todo pensamiento de justicia, y la legitimación de mecanismos de represión y de violencia institucional para el control de nuestro pueblo. Es este contexto, el que ha dictado las pautas de mi formación, y carrera profesional. De modo que, me es importante aclarar, que la comprensión de los asuntos que me propongo discutir, con sus posibles contradicciones, tiene como algunos de sus referentes la teoría social crítica, las críticas al orden capitalista, y su injerencia en la salud y salud mental. Por tanto, el abordaje parte de un entendimiento más complejo y no desde el sujeto patologizado. Dicho eso me propongo: a) Exponer el marco de los derechos humanos $y$ el derecho a la salud desde los organismos internacionales, reconociendo que existen diversas críticas a su concepción; b) Analizar brevemente el rol de la agenda global neoliberal en los derechos humanos, incluyendo la salud y salud mental; c) Discutir el impacto de la agenda global neoliberal en los procesos de patologización y deshumanización de la salud mental; d) Reflexionar en torno al rol ético-político de la Academia en la defensa de este derecho humano.

\section{Salud mental como derecho humano}

De acuerdo a la Organización de las Naciones Unidas (ONU) (2019), los derechos humanos son "derechos inherentes a todos los seres humanos, sin distinción alguna de nacionalidad, lugar de residencia, sexo, origen nacional o étnico, color, religión, lengua, o cualquier otra condición. Entre los derechos humanos se incluye el derecho a la vida y la libertad; a no estar sometido ni a esclavitud ni a torturas; a la libertad de opinión y de expresión; a la educación y al trabajo, entre otros muchos". Estos derechos están basados en cuatro (4) principios rectores: el principio de universalidad, que se refiere a que todos los derechos y libertades humanas le pertenecen a toda persona no importando procedencia, historia personal, sexo, raza, religión, creencia, preferencia sexual, o cualquier otra condición o situación. El principio de indivisibilidad plantea que todos los derechos y libertades no pueden ser divididos, fragmentados o jerarquizados. EI principio de interdependencia, por su parte, sostiene que todos los derechos y libertades humanas se encuentran entrelazados unos con otros y entre sí, asegurando que para la realización de un derecho humano es necesario la realización de otros derechos; así mismo, si es 
vulnerado un derecho se vulneran o violentan los demás. Y, por último, el principio de progresividad, el cual sostiene que todos los derechos y libertades deben ser cumplidos en su conjunto en cada momento histórico de manera constante, permanente y continua para lograr el desarrollo integro de la dignidad de la persona, prohibiéndose cualquier retroceso (Rodríguez Lozano, 2019).

Permítanme detenerme brevemente a discutir algunas críticas que me parecen importantes considerar al momento de plantear los derechos humanos para evitar cierto romanticismo que a mi entender ha acaparado este discurso. No existe una sola perspectiva de los derechos humanos. Los derechos humanos tal y como han sido formulados, tienen una fuerte influencia de la cultura occidental, pretendiendo la universalidad de los mismos. Esto ha generado amplias críticas, pues se han visto más como un discurso de imposición de la cultura occidental que como un espacio de lucha por la dignidad humana (Cordero, Palacios, Fernández, 2006). Además, en el caso de la Declaración Universal, la participación de la mayoría de los pueblos del mundo estuvo muy limitada. Solo 61 países participaron en esta Declaración, levantando el cuestionamiento de para quiénes y por quiénes se redactó. El concepto de universalidad ha sido igualmente cuestionado, pues parte del reconocimiento de la persona sin historia, cultura, raíces, y que se le atribuyen simplemente por su mera condición natural o biológica. Esta es abordada como una cuestión más específica de la cultura occidental, ocultando el elemento de lo particular y lo diverso. Hannah Arent, la filósofa y teórica política de origen judío, señalaba la transformación de los estados-nación como una de las raíces de la noción de derechos humanos. Por ejemplo, el concepto de ciudadanía enmarcada en la visión jurídica de la nacionalidad, les da la potestad a los estados a definir y reconocer una persona ciudadana con derechos versus de aquella que no es reconocida. De acuerdo con Cordero, Palacios, Fernández (2006), hay que superar la noción de ciudadanía tal y como se concibe en los países occidentales donde los derechos y libertades individuales se reconocen en la medida en que son incluidas en las leyes, sin que ello considere las condiciones sociales, económicas, culturales y políticas que permitan su máxima realización y el reconocimiento de los derechos humanos sin exclusiones. Por tanto, los derechos humanos deben trascender el orden jurídico de los países.

De otro lado, De Sousa Santos (2002), sociólogo del derecho, ha planteado que la complejidad de los derechos humanos en el marco de la globalización neoliberal, radica en 
que pueden ser concebidos como una globalización desde arriba (aludiendo a países predominantemente del norte global) o desde abajo (las comunidades y barrios, los pueblos originarios, los movimientos sociales de resistencia, aquelles que han quedado en la periferia). Es entonces cuando este proceso neoliberal y aniquilador para la humanidad que estaremos abordando más adelante pudiera revertirse si la misma se plantea de abajo hacia arriba, como un proceso contra hegemónico. Por eso la importancia de mirar con criticidad el discurso de los derechos humanos. Valdría la pena entonces preguntarnos, ¿qué implicaría esta globalización de abajo hacia arriba? ¿Qué rol pudieran jugar las instituciones académicas en este proceso? Una de sus formas pudiera ser a través de la convergencia de iniciativas y movimientos sociales, donde las universidades pudiéramos ser actores e interlocutores junto a otros sectores de la sociedad. En el sector salud, dicha convergencia implicaría el reconocimiento y reclamación del derecho a la salud y el derecho a la participación de todos y todas. O sea, un grito de reclamo del derecho a un buen vivir desde lo colectivo.

De acuerdo con Rivera (2019), profesor de derecho de la Universidad de Puerto Rico, y haciendo referencia a los trabajos de Hannah Arent y Alain Badiou, la mayor contradicción de los derechos humanos es precisamente el triunfo del capitalismo en el planeta y sus nuevas configuraciones. Para Arent (2018), la premisa de que los derechos humanos existan por virtud del simple hecho de ser seres humanos, pudiera posicionarnos a mirar las necesidades básicas como unas de carácter natural e individual y no desde una dimensión política; por eso la caridad y la filantropía han tomado fuerzas con la bendición del Estado. Ello particularmente es la esencia de análisis de este trabajo. Habiendo discutido estas críticas al marco de los derechos humanos, no quiero sonarles en clave pesimista. Al contrario, debo aclarar que ninguno de los autores y autoras citadas pretende con ello, cancelar los derechos humanos como una aspiración que debemos alcanzar todas las sociedades, y la salud como derecho humano es sin duda una agenda apremiante. Estas miradas críticas nos posicionan en un mejor estado para abordar la realidad que vivimos de una forma más consciente.

La salud como derecho humano, ha sido reconocido de forma explícita a través de diversos instrumentos internacionales. La Declaración Universal de Derechos Humanos del 1948 en su artículo 25, reconoce como pilar fundamental el derecho a la salud, el cual incluye la salud mental. El artículo 25, donde quedó consagrado este derecho ya hacen 71 años nos indica: 
Toda persona tiene derecho a un nivel de vida adecuado para la salud y el bienestar de él, así como el de su familia, incluyendo la alimentación, el vestido, la vivienda, la asistencia médica y los servicios sociales necesarios; tiene asimismo derecho a los seguros en caso de desempleo, enfermedad, invalidez, viudez, vejez u otros casos de pérdida de sus medios de sustento por circunstancias más allá de su control. (Art. 25, Declaración Universal de los Derechos Humanos, 1948).

Desde entonces, múltiples pactos, tratados y convenciones han cobijado este derecho tales como el Pacto Internacional de los Derechos Económicos, Sociales y Culturales aprobado en el 1966. Este Pacto particularmente estableció que: "Los Estados Partes en el presente pacto reconocen el derecho de toda persona al disfrute del más alto nivel posible de salud física y mental" (ONU, 1966, art. 12.2). Los Estados Partes bajo este tratado tienen el deber de cumplir con niveles mínimos de cada uno de los derechos, entre ellos:

a) la reducción de la mortinatalidad y de la mortalidad infantil, y el sano desarrollo de los niños; b) el mejoramiento en todos sus aspectos de la higiene del trabajo y del medio ambiente; c) la prevención y el tratamiento de las enfermedades epidémicas, endémicas, profesionales y de otra índole, y la lucha contra ellas; d) la creación de condiciones que aseguren a todos asistencia médica y servicios médicos en caso de enfermedad. (ONU, 1966, art. 12.2.a-d).

Otros tratados vinculados al tema del derecho a la salud son: a) la Convención sobre los Derechos del Niño, b) la Convención Internacional paralaEliminación deTodaslas Formas de Discriminación contra la Mujer, y c) la Convención Internacional para la Eliminación de Todas las Formas de Discriminación Racial, entre otros. Si nos fijamos, estas medidas parten de la definición de salud como "un estado de completo bienestar, físico, mental y social, y no simplemente la ausencia de enfermedad (OMS, 2019). Es importante reconocer que, aunque contamos con la existencia de estos tratados que recogen los derechos humanos, los mismos son productos de luchas y movimientos sociales conquistados a lo largo de la humanidad, no fueron dádivas del Estado ni de los organismos internacionales.

El derecho a la salud es uno inclusivo que abarca de igual manera la salud mental (ONU, 2018). Cada persona pudiera definir la salud mental de forma muy diferente. De acuerdo a la Organización Mundial de la Salud (2018), la salud mental “....es un estado de bienestar en el que la persona realiza sus capacidades y es capaz de hacer frente al estrés normal de la vida, de trabajar de forma productiva y de contribuir a su comunidad" 
(párr. 2). Si miramos este concepto a través de la historia, la salud mental también es definida como un constructo social que ha ido transformándose de acuerdo a los contextos políticos, económicos, sociales y culturales. Bien lo planteaba Foucault (1987), la salud mental o la carencia de ella no está ajena a la historia o a la cultura en la que surge. Esto es, no solo debemos preguntarnos por la enfermedad mental, sino por las condiciones que rodean su advenimiento en la sociedad. Desde las sociedades primitivas, que creían que las condiciones de salud mental eran producidas por maleficios, o causas sobrenaturales, luegoconlallegadadel cristianismo, se concebían como una enfermedad permanente producida por demonios para lo cual había que exorcizar o quemar en la hoguera. Ya en épocas más recientes, y con el advenimiento de la psiquíatra y las aportaciones de las ciencias sociales, surgen las nuevas clasificaciones de condiciones de salud mental y con ellas los tratamientos, centradas en el sujeto enfermo.

Esta historia dio paso a crasas violaciones de derechos humanos, exclusión, maltrato, marginación perpetradas en nombre de la medicina y el paradigma biomédico reduccionista a personas con diversidad funcional $o$ aquellas que se alejaban de los códigos sociales, políticos, y culturalmente predominantes (ONU, 2017). De acuerdo al pasado relator especial sobre derecho a la salud de las Naciones Unidas, Paul Hunt (2005), "...la salud mental es uno de los componentes más manifiestamente descuidados del derecho a la salud" (ONU, 2005, p. 5). Basta con mirar algunos datos a nivel mundial que lo evidencian. Alrededor de 450 millones de personas sufren de trastornos que afectan su diario vivir, siendo la mitad de las condiciones presentadas en la vida de una persona antes de los 14 años. Cada año, 800,000 personas se suicidan, siendo miles adicionales las que a diario intentan hacerlo. Es importante resaltar que en el $79 \%$ de los suicidios ocurre en países de ingresos bajos y medianos. Además, para el 2016, fue la segunda causa de muerte entre jóvenes de 15 a 29 años en todo el mundo, jóvenes que pudieran ser nuestros universitarios y universitarias (OMS, 2019). Ello es así no solo por la insuficiencia de servicios o medicamentos accesibles, sino porque si concebimos la salud mental como un derecho humano, ello requiere mirar las conexiones existentes entre esta y la desigualdad, así como las dificultades para satisfacer otros derechos humanos. Por tanto, la violación de derechos y por, sobre todo, del derecho a tener derechos, socaba la posibilidad de gozar de salud mental. Entonces, a bien lo ha establecido la ONU (2017) “...la salud mental no es meramente 
un problema médico o de salud; es en buena medida una cuestión de derechos humanos, dignidad y justicia social" (p.12). Lo que trato de plantear es que la salud está determinada por las condiciones en que viven las personas, que a la vez son definidas y creadas por políticas sociales y económicas en los países y más allá de sus fronteras, que impiden la realización plena de los derechos (Bernardini, 2012).

Esto me lleva a transitar al marco de los determinantes sociales de la salud. En el 2004, la Organización Mundial de la Salud expresó la intención de crear una comisión mundial sobre los determinantes sociales de la salud. La misma tenía el propósito de “...avanzar la agenda en favor de la equidad y fortalecer el apoyo de la Organización a los Estados Miembros en la implementación de enfoques integrales sobre los problemas de salud, incluidas sus raíces sociales y ambientales" (OMS, 2005, p. 43). En el 2008, esta comisión definió los determinantes sociales de la salud, incluyendo la salud mental como aquellas condiciones en que las personas nacen, viven, trabajan y envejecen, incluyendo el sistema de salud (OMS, 2008). El diagrama presentado fue el resultado de este trabajo rendido en un informe donde detalla los determinantes estructurales de las desigualdades en salud, y los determinantes intermedios de la salud.

\section{Figura 1: Marco conceptual de la Comisión de los Determinantes Sociales de la Salud}

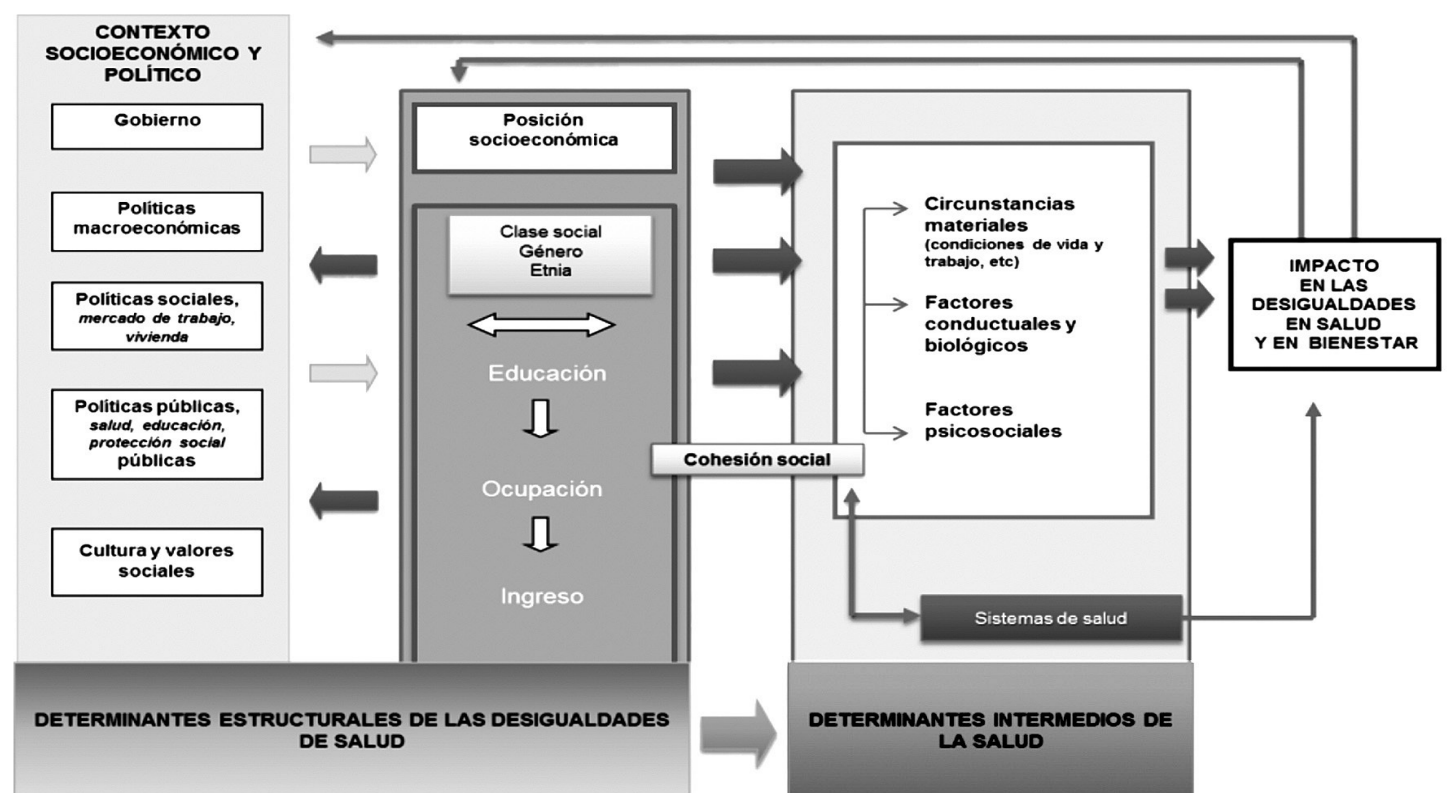

Fuente: Solar O, Irwin A. (2010). A conceptual framework for action on the social determinants of health. Social Determinants of Health Discussion Paper 2 (Policy and Practice). Geneva; World Health Organization. Versión español Organización Panamericana de la Salud/OMS (s/f). 
En el primero, se detallan los elementos relacionados al contexto socioeconómico y político tales como gobierno, políticas macroeconómicas, políticas sociales, mercado de trabajo y vivienda, políticas públicas educación, salud, protección social, cultura y valores sociales. Estos directamente relacionados con la posición socioeconómica, clase social, género, etnia, educación, ocupación, e ingresos. Estos determinantes estructurales producen los determinantes intermedios de la salud que son las circunstancias materiales como condiciones de vida y trabajo de las personas, los factores conductuales y biológicos, y los factores psicosociales, teniendo así un impacto en las desigualdades en salud y en el bienestar de la ciudadanía. El sistema de salud tiene un rol igualmente determinante en la desigualdad en salud que viven las personas.

Aunque son múltiples las críticas y debates que se han generado alrededor del Informe de la Comisión sobre los Determinantes Sociales de la Salud, de acuerdo a la investigadora Birn (2009), este informe realiza 3 contribuciones fundamentales: a) da mayor legitimidad a los determinantes sociales, haciendo una contribución a la medición y monitoreo de la inequidad en salud; b) discute dimensiones mundiales que inciden en las inequidades sociales en salud, y c) reconoce el rol de los sistemas públicos de salud como determinante. Sin embargo, dos de las grandes limitaciones identificadas y que han sido materia de discusión por expertos de la medicina social y la salud colectiva, son la falta de análisis sobre el contexto político de los problemas financieros y organizacionales de la OMS en las pasadas décadas (Birn, 2009); Navarro, 2009), así como la ausencia del análisis sobre el poder, quienes lo perpetúan y su contextualización histórica. O sea, un informe carente de la dimensión política y de un análisis sobre las causas de las causas (Birn, 2009).

A pesar de estas limitaciones, es importante señalar que la Comisión reconoció la desigualdad en salud como el principal problema mundial y expresó que sus causas no se deben a factores biológicos o genéticos, estilos de vida, servicios de salud o riqueza general del país (Benach, Vergara \& Muntaner, 2008, p. 35). Al contrario, este informe detalla algunos factores tales como: a) las malas condiciones laborales, b) no poseer una vivienda digna, c) no disponer de agua limpia, d) la falta de protección y servicios sociales, e) falta de recursos económicos, f) falta de recursos educativos, y g) falta 
de oportunidades necesarias para una vida saludable y digna. Aunque adolece de explicación sobre los mecanismos de poder que generan estos factores, podemos afirmar que la carencia de salud y salud mental es producto de la violación histórica de derechos económicos y sociales que posicionan a personas a condiciones de vulnerabilidad. De acuerdo al Informe Anual del Alto Comisionado de las Naciones Unidas para los Derechos Humanos (2017) el no tener políticas “...que tome en consideración estos y otros factores determinantes de la salud mental constituye, asimismo, un importante obstáculo a la realización del derecho a la salud mental" (ONU, 2017 , p. 6). Dicho esto, me parece entonces importante moverme al próximo punto de esta discusión; la globalización neoliberal y su impacto en las políticas llamadas a garantizar los derechos humanos, incluyendo la salud y la salud mental.

\section{La globalización neoliberal y los derechos humanos}

La globalización neoliberal es una configuración del sistema económico capitalista, o nuevo orden económico mundial, que nace entre la década de los 60 y 70 . Su arquitecto principal lo fue el Dr. Milton Friedman, profesor de economía de la Universidad de Chicago quien promovía la primacía de la libre competencia y la libertad del mercado. Este orden económico hegemónico que ha tomado mucha fuerza desde entonces, no sólo actúa en el terreno de las políticas de mercado como se planteaba en sus inicios, sino que incide de igual manera en el terreno económico, político, social y cultural de los países (Guinsberg, 2004). Esta promueve un mercado enfocado en la creación y acumulación de riqueza de parte de un sector pequeño a costa de la desposesión de la gran mayoría, la canalización de mano de obra barata, la flexibilización del mercado de trabajo, el desmantelamiento y la privatización del sector público y consigo los servicios llamados a garantizar los derechos humanos como lo es la salud, la educación, la vivienda, el trabajo, la vida digna, entre otros (Guinsberg, 2004; RiveraDíaz, 2018).

Por las pasadas cuatro décadas, y bajo la lógica de este orden económico neoliberal, muchos países, incluyendo a Puerto Rico, han enfrentado políticas que han ido transformando los derechos humanos en mercancías rentables, convirtiendo a su vez a las personas en consumidores y compradores de sus derechos. Solo que quienes podrán adquirirlos serán los que tienen el capital para su consumo, población que cada vez ha venido 
achicándose. También, se han promovido programas de ajuste o control fiscal, imponiendo recortes drásticos en el área de servicios sociales, educativos, nutricionales, de vivienda, y seguridad social y pensiones, todos reconocidos como derechos humanos (Rivera-Díaz, 2018). El Estado ha recortado en las nóminas y salarios de empleados del sector público, provocando despidos masivos, y altas tasas de desempleo y pobreza. De esta forma, ha socavado el estado de derechos adquiridos en luchas sindicales y obreras, actuando contra los sistemas de retiro y el despido en masas de los empleados del sector gubernamental. Es a esto lo que David Harvey, geógrafo y antropólogo marxista, denomina la acumulación mediante desposesión (Harvey, 2004). El despojo de nuestros derechos bajo la bandera de la austeridad y la mercantilización. Entonces, esta mercantilización de los derechos humanos, opera como una sangría devastadora, pauperizando las condiciones de vida, de salud y salud mental de la población al punto que, para algunos estudiosos, es la mayor enfermedad del planeta (Benach, Vergara \& Muntaner, 2008).

Esta desigualdad se asocia con mayores niveles de desempleo, trabajos precarios e inestabilidad que provocan a su vez altos niveles de estrés, patrones de alimentación no saludables, pobre acceso a la vivienda, cronicidad de condiciones de salud física y mental, menor acceso y disponibilidad a servicios de salud y salud mental, pobre acceso a la educación, entre otros (Labonté \& Stuckler, 2016).

\section{Vulnerabilización de la salud mental ante la agenda neoliberal en los derechos humanos}

En el espacio de la salud, la agenda neoliberal encontró un atractivo mercado. Colocó a esta en el ámbito privado como responsable de las actividades ligadas al cuidado de la salud y desvinculó al Estado como garante de este derecho a la población (Laurell, 1992; 1994). Las reformas neoliberales en el sector salud emprendidas en muchos países en las décadas del 80 y 90 se enfocaron en tratar los problemas económicos y estéticos de los sistemas de salud tales como: a) la necesidad de poner límites a los gastos del sector de la salud, b) aumentar la presencia del sector privado integrando opciones privadas para el financiamiento y prestación de los servicios de salud, c) dividir las funciones de financiamiento, adquisición y prestación de servicios; d) descentralizar, e) entre otros (OMS, 2005). Como consecuencia, dichas reformas propiciaron una reducción significativa del rol del 
Estado en el sector salud y en otras áreas neurálgicas de la sociedad que inciden directamente en la salud de la población. Entonces, tenemos personas enfrentando angustias, malestar emocional producto de todas estas violaciones frente a un Estado que no tiene la capacidad de responderle.

Además, la concepción de la salud fue transformada por esta ideología, pasando a ser una responsabilidad individual y privada, ello por la noción de que el Estado era ineficiente para proveer los servicios. De acuerdo con Asa Cristina Laurell (1992), si hacemos un análisis de esta ideología desde una perspectiva crítica, su finalidad era la de ubicar a la salud como actividad privada con el objetivo de convertirla en un nuevo campo de acumulación capitalista. Bien lo dijeron Stolkiner y Ardila (2012) que para lograr los cambios que requiere la globalización neoliberal, esta colocará la vida como objeto mercantil, donde la construcción de los problemas de salud se remplaza por la visión corporativa de la enfermedad.

La salud mental igualmente se vio atravesada por esta agenda neoliberal, aplicando la privatización, el desmantelamiento y desarticulación paulatina del cuidado de la salud mental a raíz de la lógica de reformas. Dichas reformas lograron la desvinculación del Estado, generando escasez de servicios preventivos y de inclusión social a personas con condiciones de salud mental. Esta ideología neoliberal, de la mano de la industria farmacéutica, el complejo médico industrial y las instituciones oficiales de la psiquíatra y la psicología a nivel mundial, han impulsado la epistemología atrasada de la salud mental como sinónimo de enfermedad mental. Si vemos hoy día, la mayoría de los recursos económicos, recursos humanos y de infraestructura, aunque muy limitados, están destinados a la atención médica y no a la prevención. Ello a su vez, ha llevado a la sustitución de terapias alternativas por la hipermedicalización de los síntomas, la aplicación de prácticas de patologización generando sobrepoblación en hospitales psiquiátricos, y la medicalización de los problemas sociales.

No se equivocaba Foucault (1987) cuando indicó que la 'psiquiatrización' de la vida cotidiana, si se la examinase de cerca, revelará posiblemente lo invisible del poder. Como resultado, estas prácticas que han acaparado el campo de la salud mental lo que han logrado es posicionar al modelo biomédico del complejo médico industrial el cual impone una mirada biologicista e individualista de la salud y la salud 
mental, para el control, sometimiento y exclusión de las personas y sus cuerpos. Por ejemplo, en muchos escenarios, se les culpa y penaliza a las personas por su pobre condición de salud, por no tener una cubierta de salud, por su consumo prolongado de drogas, por no asistir con regularidad a sus citas, por no mudarse del lugar contaminado en donde vive, y en otras se les despacha con la idea "de que existe una predisposición a las condiciones que padecen". En tiempos de pandemia, este discurso ha cobrado vital importancia con frases tales como "quédate en casa", "el cuidado es individual", "cuida a los tuyos". Como bien indican Molina y Tobar (2018), se le traslada a la persona la administración del riesgo para enfrentar sus enfermedades. A ver, ¿cuántas veces nos han dicho que para preservar la salud mental debemos: a) tener una actitud $y$ pensamientos positivos; b) hacer ejercicios al menos 150 minutos a la semana; c) mantener una buena condición física; d) dormir lo suficiente; f) meditar; g) evitar el estrés; y h) buscar ayuda profesional si lo necesita? Esto, aunque pudiera tener sus beneficios, lo que ha hecho es reforzar el enfoque biomédico de la salud que ha servido de andamiaje al mantenimiento de las reformas neoliberales en dicho sector. Ese modelo biomédico insiste clasificarnos entre enfermos y sanos, entre normales y no normales, entre funcionales o disfuncionales, dejando afuera los determinantes sociales y las estrategias multisectoriales que bien pudieran atender las dimensiones estructurales de la salud (Molina \& Tobar, 2018).

Tenemos que estar conscientes que, aunque los resultados y el dolor de la desigualdad que provocan dichas políticas las personas la sienten, la viven y la padecen de forma individual en la cotidianidad de sus vidas, las mismas no son un fenómeno natural producto de su falta de voluntad, de su predisposición genética, de su incapacidad para adaptarse a los cambios, de sus comportamientos de riesgos, estilos de vida o cualquier otra característica individual a la cual se le adjudique la responsabilidad de su mal (RiveraDíaz, 2018). El insistir en esta mirada individualista sin comprender que las pésimas condiciones de salud y salud mental que enfrenta la ciudadanía son producto de la persistente e histórica violación de derechos humanos, nos hace igual de transgresores y transgresoras. Dichas violaciones, que imposibilitan vivir dignamente, son injustas, innecesarias y prevenibles, y se reflejan en un sector cada vez más amplio de la sociedad. En ocasiones, estas violaciones nos posicionan como perpetuadores y perpetuadoras 
de éstas a través del señalamiento, los prejuicios, la estigmatización, el discrimen, la marginación y exclusión, deshumanizando y patologizando a quien la enfrenta.

\section{Rol de la Academia en la defensa de la salud mental como derecho humano}

El último punto que me propongo atender entonces es entorno a la pregunta: ¿cuál es el rol de las instituciones académicas para la defensa de la salud mental como derecho humano? ¿Cómo aportamos a la humanización de este derecho? La Universidad posee diversos roles, no solo en los procesos de formación profesional, intelectual, cultural y emocional de jóvenes adultos, sino también como espacio gestor de justicia social y educativa (Torres Abreu, et al., 2020). Las instituciones académicas se encuentran en un momento crucial para responder a este llamado de índole ético-político. En la Encuesta Mundial de Valores para Puerto Rico 2018 llevada a cabo por la Universidad del Sagrado Corazón y el Instituto de Estadísticas de Puerto Rico, si bien reseñara ante la prensa que el $91 \%$ de la población se consideraba bastante feliz o muy feliz, el $66.3 \%$ considera buena o muy buena la salud (Hernández, 2019).

Además, elementos vinculantes a los determinantes sociales de la salud que hemos definido, tales como: el temor a salir de la casa en la noche (54\%), la falta de interés en los asuntos políticos (52\%), la escasez de comida o percepción de inseguridad en su hogar (33\%), entre otros, son algunos elementos que se enfatizan y que merecen nuestra atención. Por otro lado, pese a que existe la falta de confianza en instituciones como el gobierno (-28\%), las universidades son las que generan un mayor nivel de confianza en nuestra sociedad con un 79.8\% (Hernández, 2019). Este dato es importante subrayarlo pues, de acuerdo al estudio, las instituciones académicas gozan de la confianza y credibilidad para dar respuestas a la construcción de una sociedad que aspire al bien colectivo $\mathrm{y}$, por tanto, a la defensa y expansión de los derechos humanos. No debemos dudar, que esta confianza va acompañada de una expectativa social de hacer algo que trascienda las verjas y portones de nuestras instituciones.

¿Cómo encaminar un proyecto ético político desde las instituciones académicas que aporte en la defensa y expansión de los derechos humanos, incluyendo la salud y la salud mental? Las respuestas a esta pregunta son complejas y no pretendo aquí dar por contestada esta pregunta en su totalidad. Sin embargo, me parece que es importante compartirles algunas reflexiones que puedan ayudarnos 
a dinamizar, trazar y pavimentar ese camino. Para ello, abordaré propuestas preliminares en tres áreas a saber: a) investigación y producción de conocimiento, b) Academia al servicio de la comunidad, c) prácticas institucionales de acompañamiento.

\section{a. Investigación y producción conocimiento}

Empecemos con la investigación y producción de conocimiento. En el contexto actual, no podemos negar que la educación superior ha sido foco de ataque del proyecto neoliberal de igual forma que la salud y demás derechos sociales. Este ha penetrado en las instituciones universitarias y los espacios de producción de conocimiento. Muchas de las investigaciones que se producen, se dan bajo la lógica de cumplimentación de requisitos académicos, abandonando el objeto de estudio y el para qué hacemos investigación (Rivera-Díaz, 2019). Otros elementos que me parecen importantes mencionar como resultado de esta penetración de la lógica neoliberal en el espacio académico son: a) la precarización de las condiciones de trabajo de los académicos investigadores e investigadoras, b) el control de la agenda investigativa institucional por entes externos de financiamiento, c) la privatización del conocimiento científico por parte de grandes editoriales comerciales, d) producción y prevalencia de un conocimiento para mantener el estatus quo e invisibilización de las raíces verdaderas de las injusticias sociales, y principales productores de las condiciones de salud mental (Rivera-Díaz, 2018).

Por tanto, desde este espacio exhorto a quienes tienen injerencia en los programas académicos a que no claudiquen ante la tendencia de reducir o limitar la formación en investigación. Siempre que investigamos, partimos del cuestionamiento de que existe algo más que no está visible en la realidad inmediata que se nos presenta o en cómo esa realidad se manifiesta e impacta la vida de las personas (Rivera Díaz, 2018.). La forma en cómo surge este cuestionamiento va a depender del abordaje teórico con que miremos esa "realidad social" y las respuestas que daremos tanto en la investigación como en las intervenciones en el campo de la salud mental o en otras dimensiones de la vida. Por ejemplo, en el campo de la salud, tal como hemos discutido aquí, el modelo biomédico ha logrado cobrar un gran apoyo del complejo médico industrial que impone una mirada mercantilista, biologicista e individualista de la salud. Es por esto que, los estudios que se centran en la patologización de la cuestión social, en la caracterización y elaboración de perfiles de personas en riesgos 
a trastornos o condiciones, etc. han contado con mayor apoyo económico y académico. Tiene mucha razón Yolanda Guerra (2015), profesora de Trabajo Social de la Universidad Federal de Río de Janeiro cuando nos dice que "Cada modalidad de conocimiento nos permite una forma de apropiación del mundo" (p.129).

Los procesos investigativos sirven para visibilizar la formas injustas y opresivas de los embates del neoliberalismo que agudiza la desigualdad (Muñoz Arce, Hernández \& Veliz Bustamante, 2016) y las condiciones de salud y salud mental. La investigación nos ayuda a tener una clara comprensión de la complejidad social, sirviendo esta experiencia como un eslabón importante para proponer, diseñar, gestionar e implementar procesos de intervención justos y sensibles a nuestras poblaciones (Muñoz Arce, Hernández \& Veliz Bustamante, 2016). El acto de investigar es un acto eminentemente político cuando cumple con su propósito de contribuir al proyecto social a través no solo de la generación de un conocimiento nuevo, sino del desarrollo de un pensamiento crítico capaz de problematizar y hacer frente a los problemas sociales (Falla, 2009) generadores del malestar emocional y mental que vive la población. Para ello, entonces es necesario: a) la convergencia de disciplinas en las investigaciones sin abandonar la independencia metodológica (Falla, 2009), y b) trascender la investigación hacia una que sirva como herramienta de acompañamiento a los movimientos sociales y comunitarios que enfrentan las consecuencias de esta agenda neoliberal y revierta la desigualdad social.

\section{b. Academia al servicio de la comunidad}

La segunda propuesta va dirigida a la construcción de una Academia al servicio de la comunidad que sea capaz de accionar en los determinantes sociales de la salud y la salud mental, encaminadas al buen vivir. Ello es posible, pues como institución todavía gozamos de la credibilidad social y existe una expectativa de respuestas ante la debacle y barbarie social que enfrentamos. Por tanto, nuestra responsabilidad social es aún mayor y su misión debe dirigirse a ampliar esta discusión del lado de las comunidades.

\section{Recomendaciones:}

1. Resignificar el rol de la universidad con su responsabilidad social y la defensa, exigibilidad y expansión de los derechos humanos.

2. Fortalecimiento de la comunidad académica para combatir la 
desigualdad, la inequidad, y asumir la responsabilidad social como una práctica ética y política.

3. Desarrollar estrategias educativas en espacios comunitarios con estudiantes y docentes que aporten a la transformación de la visión mercantilista de la salud a una de derecho y bien común.

4. Implementación de estrategias de aprendizaje basadas en servicio con proyectos comunitarios interdisciplinarios que incidan en los determinantes sociales de la salud y la salud mental.

5. Desarrollo de investigaciones basadas en la comunidad 0 aplicación de metodologías investigativas de acompañamiento como lo es la investigación acción participativa que propicien cambios a corto, mediano y largo plazo.

6. Participar activamente en los debates de las diversas problemáticas o retos sociales, económicos y políticos que enfrentan los grupos de la sociedad para junto a estos, diseñar soluciones prácticas que aporten al desarrollo, la justicia social y el alcance de la salud como un derecho humano.

7. Problematizar junto a sectores académicos y comunitarios sobre los mecanismos que producen la desigualdad, particularmente mirando las relaciones y distribución del poder en la sociedad y aportar en la búsqueda de alternativas que propicien la construcción de una sociedad más equitativa.

8. Creación de centros de servicios psicosociales y proyectos productivos permanentes para el desarrollo comunitario. Estos centros pueden estar en las instalaciones universitarias 0 fuera de ellas, al interior de los espacios comunitarios.

9. Asesoramiento y actividades realizadas por la universidad para beneficio de la comunidad, grupos sociales $u$ organizaciones que atienden los determinantes sociales de la salud.

10. Educación sobre defensa y expansión de los derechos humanos a la comunidad y grupos sociales. Ello acompañado del desmantelamiento de prácticas filantrópicas que no permiten que las personas se conciban como ciudadanos y ciudadanas con derechos y aporte a la construcción de una sociedad democrática. Los derechos humanos son un deber no un favor, ni pueden estar a expensas de la caridad y la filantropía. 
11. Integración de activistas sociales, organizaciones de base comunitaria y profesionales en el diseño de currículos académicos que respondan a las necesidades y retos sociales emergentes.

12. Promover políticas de equidad en el acceso a la educación universitaria, reconociendo la educación como un derecho humano fundamental que tendrá implicaciones positivas a su vez en la consecución de otros derechos como la salud mental.

13. Capacitar al estudiante de experiencias que le doten como conocedor/a y defensor/a de la justicia social y los derechos humanos a través de actividades dentro y fuera del salón de clases, vínculos permanentes con escenarios, grupos $y$ alianzas, elaboración de trabajos que tengan injerencia directa en procesos locales einternacionales de reconocimiento, defensa, promoción, ampliación de derechos humanos. Parte de estas experiencias debe integrar procesos evaluativos reflexivos de construcción y reconstrucción de comportamientos, actitudes, percepciones, tanto de estudiantes como de profesores/as.

\section{c. Prácticas institucionales de acompañamiento}

Con relación a la tercera propuesta sobre prácticas institucionales de acompañamiento estaré considerando prácticas para con el personal y con el estudiantado. Para esto, me parece importante mirar los conceptos esenciales que promueven las Universidades Promotoras de la Salud (UPS), movimiento internacional apoyado por la Organización Mundial de la Salud enfocado en trabajar con el desarrollo de entornos y prácticas saludables dirigidas a conocer, apoyar, y fortalecer estilos saludables entre el personal, profesorado y estudiantes (Bravo, Cabieses, Zuzulich, Muñoz, \& Ojeda, 2013). De acuerdo a Muñoz y Cabiases (2008) las universidades:

...tienen el potencial para influir positivamente en la vida y condición de salud de sus miembros, protegiéndolos y promoviendo su bienestar y pueden liderar $y$ apoyar procesos de cambio en la comunidad externa, a través de sus políticas y prácticas. Además, al ser organizaciones extensas y complejas, las personas que ahí trabajan o estudian les dedican gran parte de su tiempo, y algunas, gran parte de su vida, por lo que los determinantes de la salud están regidos en gran medida por las condiciones o características sociales en las cuales esas 
personas viven, estudian o trabajan en la universidad (p.145).

Con relación al estudiantado, una investigación realizada con una muestra de 450,000 estudiantes universitarios en Estados Unidos reveló que la ansiedad, la depresión y los ataques de pánico son las principales condiciones de salud mental entre esta población (Oswalt, Lederer, Chestnut, Day, Halbritter, \& Ortiz, 2018). Interesantemente, este estudio también reveló que los estudiantes estaban más dispuestos a utilizar los servicios universitarios de salud mental, lo que sugiere un rol más activo de parte de las instituciones en salvaguardar el bienestar de sus estudiantes (Oswalt, Lederer, Chestnut, Day, Halbritter, \& Ortiz, 2018). Esta realidad no se aleja de Puerto Rico, y se agrava aún más con la falta de acceso y disponibilidad de servicios de salud mental para la población juvenil $y$ adultos mayores, particularmente aquellos/as bajo la Reforma de Salud del Gobierno, nuestros jóvenes más pobres y aquellos que no tienen ninguna cubierta de salud. El Dr. Eduardo Lugo, profesor de psicología del Recinto de Mayagüez de la Universidad de Puerto Rico expresaba en una red social en el 2019 su preocupación ante la cantidad de universitarios y universitarias con necesidad de servicios psicológicos en la zona en la que ubica el campus y sin un solo psicólogo que acepte la Reforma de Salud del Gobierno de Puerto Rico. Coincide en que la pobreza y la desigualdad en el país son barreras para la atención del derecho a la salud mental.

Los docentes y el personal en instituciones académicas, al igual que los y las estudiantes, no están exentos de vivir las condiciones sociales que vulnerabilizan su salud mental. Un estudio realizado en el 2014 con docentes en Colombia reveló que las condiciones de trabajo, los ambientes laborales y las cargas académicas se suman a los estresores que determinan el disfrute de una buena salud mental (Villamizar, Padilla, \& Mogollón, 2014). Entonces, ¿qué propuestas pudiéramos considerar que nos permitan reconocer y abogar por la salud mental como derecho humano desde nuestras instituciones para con el estudiantado, pero también para con nuestros docentes y toda la comunidad universitaria?

\section{Recomendaciones:}

1. Fomentar el desarrollo de programas que se centren en la detección temprana de problemas de salud mental o condiciones de vida de nuestros estudiantes y personal docente y no docente que les vulnerabilizan, 
previniendo así su agudización y complicaciones con la vida universitaria. Las universidades se convierten para muchos estudiantes como el único lugar donde pueden tener acceso a servicios de salud mental a través de las propuestas curriculares, acceso a material educativo, grupos de apoyo, y servicios individualizados.

2. Fomentar el desarrollo del liderato estudiantil en alfabetización en salud y en los determinantes sociales que inciden en ella.

3. Explorar y diseñar estrategias de prevención y apoyo que satisfagan las necesidades más apremiantes que enfrenta la comunidad universitaria. Dichas estrategias deberán estar diseñadas desde una perspectiva de género.

4. Ofrecimiento o canalización de servicios sociales, servicios médicos, y de salud integral a toda la comunidad universitaria.

5. Ofrecimiento de servicios educativos, entre pares o de mentoría académica e investigativa que sirva de acompañamiento y apoyo al estudiantado, logrando mayores niveles de retención, graduación y, por ende, satisfacción profe- sional entre egresados.

6. Realizar evaluaciones comprensivas sobre el estado de situación de los y las estudiantes y personal que permita a las instituciones identificar los retos apremiantes que enfrentan en áreas de salud, salud mental, recursos económicos, etc.

7. Fomentar el desarrollo de colectivos de trabajo interdisciplinarios con objetivos para la actuación en los determinantes sociales de la salud de la comunidad universitaria.

8. Transversalizar la formación en la promoción de la salud y los derechos humanos en los currículos de los programas académicos.

9. Propiciar la transformación del rol de los y las estudiantes en la construcción de espacios académicos necesarios para la promoción de la salud.

10.Propiciar espacios de diálogo y actividades que ayuden a deconstruir colectivamente las nociones estigmatizantes de la salud mental. De esta forma, humanizaremos el dolor, la angustia, el malestar que nos provocan los cambios sociales y económicos tan violentos que enfrentan nuestros y nuestras 
jóvenes, y la sociedad en general.

11.Implementar una cultura de derechos humanos en la educación. Para lograrlo, la Academia debe de evaluar varios aspectos: a) políticas y normativas, b) currículos de enseñanza, c) misión y visión, d) planes estratégicos, e) proyectos de gerencia académica. No basta con integrar el tema de los derechos humanos en los cursos o crear cursos de derechos humanos. En cuanto a las políticas y normativas, es importante que la misión y visión de los departamentos o unidades se articulen dentro de esta perspectiva, haciéndolo explícito a través de las políticas, estructuras organizacionales, procesos de personal y recursos humanos, planes de desarrollo, toma de decisiones y procesos de participación, y evaluación de estudiantes y personal docente.

\section{Reflexiones finales}

Para finalizar, es necesario recobrar el modelo de universidad comprometido con el desarrollo social y alejarnos del modelo neoliberal corporativo de la universidad que concibe al estudiante como cliente consumidor y la universidad como instrumento para la realización personal y demandas del sector productivo. A diferencia de este, el primer modelo concibe la universidad como un instrumento para fines colectivos, para el desarrollo y el bien común (Espinoza, 2017). El primero nos llevará a la construcción de un país más democrático, equitativo y justo. El segundo, logrará la realización académica de los que privilegiadamente han accedido a ella. Nuestro fin como instituciones académicas no es solo preparar la nueva generación de profesionales o la construcción de conocimiento. Si ello, no va de la mano con el compromiso genuino en el desarrollo de ciudadanos y ciudadanas solidarios, solidarias y sensibles con los derechos humanos y la justicia social, estamos violentando nuestra responsabilidad ético política.

Agradecimiento: La autora agradece la colaboración de Natalie Álamo y Randy Tejada, asistentes de investigación en el Centro de Investigaciones Sociales de la Universidad de Puerto Rico, en la identificación de bibliografía para la realización de este artículo. 


\section{Referencias}

Arent, Hannah. (2018). La libertad de ser libres: un ensayo inédito. Taurus.ISBN:978-84-306-2231-3

Benach, J., Vergara, M., \& Muntaner, C. (2008). Desigualdad en Salud: La mayor epidemia del siglo XXI. Papeles: 103, 29-40. https://www. fuhem.es/media/ecosocial/file/Cohesión\%20 Social/Desigualdad,\%20pobreza\%20 y\%20exclusión/desigualdad\%20en\%20 salud BENACH\%2OVERGARA\%20Y\%20 MUNTANER.pdf

Bernardini-Zambrini, D. A. (2012). El día mundial de la salud, los determinantes sociales y las oportunidades. Revista Peruana de Medicina Experimental y Salud Pública, 29 (2), 287-288. https:// www.scielosp.org/scielo.php?script=sci arttext\&pid=S1726-46342012000200022\&lng =es\&nrm=iso\&tlng=es

Birn, Anne-Emmanuelle. (2009). ¿Politizándolo o puliéndolo? Subsanar las desigualdades en una generación: alcanzar la equidad sanitaria actuando sobre los determinantes sociales de la salud. Medicina Social, 4(3), 189-201.

Bravo, P., Cabieses, B., Zuzulich, M., Muñoz, M., \& Ojeda, M. (2013). Glosario para universidades promotoras de la salud. Revista de Salud Pública, 15 (3), 465- 477. https://www.scielosp. org/pdf/rsap/2013.v15n3/465-477

Cordero Ramos, N., Palacios

Esteban, J., \& Fernández Martín, M. (2006). Trabajo social y Derechos humanos: Razones para una Convergencia. Acciones

e Investigaciones Sociales,

1. https://dialnet.unirioja.es/servlet/ articulo? codigo $=2002316$

Declaración Universal de Derechos Humanos. Adoptada y proclamada por la Asamblea general en su resolución 217 A (III), de 10 de diciembre de 1948. Naciones Unidas. https://www. ohchr.org/EN/UDHR/Documents/UDHR Translations/spn.pdf

De Sousa Santos, B. (2002). Hacia una concepción multicultural de los derechos humanos. El Otro Derecho, 28. http://www.uba. ar/archivos ddhh/image/Sousa\%20-\%20 Concepción\%20multicultural\%20de\%20 DDHH.pdf

Espinoza, O. (2017). Neoliberalismo y educación superior en Chile: una mirada crítica al rol desempeñado por el Banco Mundial y los "Chicago Boys". Laplage em Revista, 3 (3), 93-114. http://www.laplageemrevista.ufscar.br/index. php/lpg/article/view/378

Falla Ramírez, U. (2009). Reflexiones sobre la investigación social y el trabajo social. Tabula Rasa, 10, 309-325. Universidad Colegio Mayor de Cundinamarca, Colombia.

Foucault, M. (1987). El nacimiento de la clínica. Una arqueología de la mirada médica. Siglo XXI.

Guerra, Y. (2015). Trabajo Social: Fundamentos y Contemporaneidad (4ta ed.). Colegio de Asistentes Sociales o Trabajadores Sociales de la Provincia de Buenos Aires. 
Guinsberg, E. (2004). La salud mental en el neoliberalismo. Plaza y Valdés S.A.

Harvey, David. (2004). El "nuevo" imperialismo: acumulación por desposesión. Socialist Register 2004, 99-129. Accedido:

http://biblioteca.clacso.edu.ar/clacso/ se/20130702120830/harvey.pdf

Hernández Acosta, J. (2019). Encuesta Mundial de Valores para Puerto Rico: 2018. Instituto de Estadísticas de Puerto Rico. https://estadisticas.pr/en/media/3286

Labonté, R., \& Stuckler, D. (2016). The rise of neoliberalism: how bad economics imperils health and what to do about it. Journal of Epidemiology Community Health, 70, 312-318. Doi:10.1136/jech-2015-206295.

Laurell, A. (1992). Estado y política social en el neoliberalismo.

Friedrich Ebert Stiftung.

Laurell, A. (1994). La reforma del Estado y la política social en México. Nueva Sociedad, 164, 146-158. https://nuso.org/media/articles/ downloads/2818 1.pdf

Molina, C., \& Tobar, F. (2018). ¿Qué significa Neoliberalismo en salud? O que o Neliberalismo significa em saúde? RevlISE, 12, 65-73. https://www.redalyc.org/ latsRepo/5535/553557482007/553 557482007.pdf

Muñoz Arce, G., Hernández Mary, N., \& Veliz Bustamante, C. (2016). Ponencia: Articulación investigación e intervención en trabajo social. Aportes a la construcción de conocimientos disciplinar. Debates sobre el Trabajo Social y las Ciencias Sociales. Su implicancia en el contexto actual. Santiago, Chile: Universidad Alberto Hurtado.

Muñoz, M., \& Cabiases, B. (2008). Universidades y promoción de la salud: ¿cómo alcanzar el punto de encuentro? Revista Panamericana de Salud Pública, 24 (2), 139-146.

Navarro, V. (2009). "What We Mean by Social Determinants of Health," Global Health Promotion, 16 (1), 5-16.

Organización de las Naciones Unidas. (1966). Pacto Internacional de Derechos Económicos, Sociales y Culturales. Asamblea General, resolución $2200 \mathrm{~A}(\mathrm{XXI}), 16$ de diciembre de 1966. https://www. ohchr.org/Documents/Professionallnterest/ cescr SP.pdf

Organización de las Naciones Unidas. (2005). Los derechos económicos, sociales y culturales. Informe del relator especial sobre el derecho de toda persona al disfrute del más alto nivel posible de salud física y mental, Sr. Paul Hunt. Consejo Económico y Social. Comisión de Derechos Humanos. 14 de febrero de 2005. https://documents-dds-ny.un.org/ doc/UNDOC/GEN/G05/108/96/PDF/ G0510896.pdf?OpenElement 
Organización de las Naciones Unidas. (2017). Salud mental y derechos humanos. Informe anual del Alto Comisionado de las Naciones Unidas para los Derechos Humanos. Asamblea General. 31 de enero de 2017.

https://webcache.googleusercontent.com/ search?q=cache:XSOD2RuYhfMJ:

https://digitallibrary.un.org/

record/861008/files/A HRC 34 32-ES.

$\mathrm{pdf}+\& \mathrm{~cd}=2 \& \mathrm{hl}=\mathrm{en} \& \mathrm{ct}=\mathrm{clnk} \& \mathrm{gl}=$

pr\&client=safari

Organización de las Naciones

Unidas. (2018). La salud mental es un derecho humano.

24 de mayo de 2018. https:// www.ohchr.org/SP/NewsEvents/Pages/ MentalHealthlsAhumanright.aspx

Organización de las Naciones Unidas (ONU). (2019). Derechos humanos. https://www.un.org/es/ sections/issues-depth/human-rights/index. html

Organización Mundial de la Salud. (2005). Acción sobre los factores sociales determinantes de la salud: aprender de las experiencias anteriores. Documento de información preparado para la Comisión sobre Determinantes Sociales de la Salud. Marzo de 2005. Secretaria de la Comisión sobre Determinantes Sociales de la Salud. https://www.who.int/social_ determinants/resources/action sp.pdf

Organización Mundial de la Salud. (2008). Subsanar las desigualdades en una generación:
Alcanzar la equidad sanitaria actuando sobre los determinantes sociales de la salud. Informe final de la Comisión OMS sobre Determinantes Sociales de la Salud. 28 de agosto de 2008. https://www.who.int/social determinants/ final report/media/csdh report wrs es.pdf

Organización Mundial de la Salud. (2018). Salud mental: fortalecer nuestra respuesta. 30 de marzo de 2018. https://www.who.int/es/ news-room/fact-sheets/detail/mental-healthstrengthening-our-response

Organización Mundial de la Salud. (2019). Suicidio, datos y cifras. 2 de septiembre de 2019. https:// www.who.int/es/news-room/fact-sheets/ detail/suicide

Organización Panamericana de la Salud/OMS. (s/f). Determinación social del procesos salud enfermedad. Tercer Congreso Nacional de Educación en Salud Pública. https://www.paho.org/mex/index. php?option=com docman\&view=download\& category slug=technicaldocumentation\&alias $=1036-950$ amesp\&ltemid $=493$

Oswalt, S., Lederer, A., ChestnutSteich, K., Day, C., Halbritter, A., \& Ortiz, D. (2018). Trends in college students' mental health diagnosis and utilization of services. 2009-2015. Journal of American College Health. https:// www.tandfonline.com/doi/full/10.1080/074484 81.2018 .1515748 
Rivera, P. (2019). Consideraciones para un sistema de derechos humanos al revés. 80 grados, 11 de enero de 2019.

http://www.80grados.net/author/ pedrojoseriverarivera/

Rivera-Díaz, M.. (2018). Derechos humanos en salud y trabajo social en Puerto Rico: un ensayo reflexivo para una propuesta de acción. Publicaciones Puertorriqueñas.

Rivera-Díaz, M. (2019). La investigación como herramienta socio política para el proyecto de emancipación social desde el Trabajo Social. (ponencia central). En Mellizo Rojas, W. (ed.). Proyecciones profesionales, académicas y de investigación para el trabajo social en América Latina y el Caribe ante el desafío de la crisis mundial. XXII Seminario Latinoamericano y del Caribe de Trabajo Social. ALAEITS, Conets: Bogotá. ISBN 978-958-96066. https:// conetsco.org/wp-content/uploads/2019/09/ PonenciasCentrales.pdf

Rodríguez Lozano, L. (2019). Los derechos sociales y los principios de universalidad, progresividad, indivisibilidad e interdependencia. Perfiles de las Ciencias Sociales, 6 (11), 243258. Http://revistas.ujat.mx/index.php/ perfiles, México, UJAT.

Solar, O., \& Irwin, A. (2010). A conceptual framework for action on the social determinants of health. Social Determinants of Health Discussion Paper 2 (Policy and Practice). Geneva, World Health Organization.

Stolkiner, A. \& Ardila, S. (2012). Conceptualización de la salud mental en las practices: consideraciones desde el pensamiento de la medicina social/salud colectiva latinoamericanas. Vertex-Revista Argentina de Psiquiatría. http://www. psi.uba.ar/academica/carrerasdegrado/ psicologia/sitios catedras/obligatorias/066 salud2/material/unidad1/subunidad 1 1/ stolkiner ardila conceptualizando la salud mental.pdf

Torres Abreu, A., Rivera Bonilla, I., Molina Carrasquillo, R., Marcano Medina, J., Rodríguez Rodríguez, L., Rivera García, M., Cruz McDougall, V.,. y Nina, D. (2020). Brecha digital, aprendizaje y salud mental: Experiencias y retos del estudiantado de la Universidad de Puerto Rico en Humacao (UPRH) ante el COVID-19. Universidad de Puerto Rico en Humacao, Instituto Transdisciplinario de Investigación-Acción Social (ITIAS).

Villamizar, D., Padilla, S., \& Mogollón, O. (2014). Contexto de la salud mental en docentes universitarios. Un aporte a la salud pública. CES Salud Pública, 6, 146-159 